\title{
Metamaterial Coherent Light Absorption - The Time-reversed Analogue of the Lasing Spaser
}

\author{
J. Zhang, K. F. MacDonald", and N. I. Zheludev \\ Optoelectronics Research Centre and Centre for Photonic Metamaterials, University of Southampton, Southampton, SO17 1BJ, UK \\ *kfm@orc.soton.ac.uk
}

\begin{abstract}
We experimentally demonstrate a new coherent absorption phenomenon, through which a planar photonic metamaterial may resonantly absorb $100 \%$ of incident light. The effect is a timereversed analogue 'Lasing Spaser' action.

OCIS codes: (160.3918) Metamaterials; (240.6680) Surface plasmons; (030.1670) Coherent optical effects
\end{abstract}

We demonstrate for the first time that a planar photonic metamaterial - a single layer of nanostructured metal less than one tenth of a wavelength thick - can resonantly absorb all of the light incident on it. The phenomenon relies on a coherent interaction and constitutes the time-reversal counterpart of 'lasing spaser' action.

The effect is a metamaterial analogue of the recently reported coherent perfect absorption phenomenon observed in a bulk silicon optical resonator [1]. This narrowband bulk resonator effect arises from the tailored interplay of interference and absorption between counter-propagating beams in a dissipative medium and may be understood as the time-reversed counterpart of conventional lasing: Time-reversal symmetry applied to a laser at threshold dictates that the gain medium should be replaced by an equivalent absorbing medium which will perfectly absorb all incoming light with the same field pattern as that radiated by the laser.

Here we show that a similar effect may be observed in a single sub-wavelength metallic metamaterial film exploiting plasmonic resonant absorption (Fig. 1). This metamaterial implementation of coherent perfect absorption represents the time-reversed analogue of the Lasing Spaser - a low-divergence, coherent source of electromagnetic radiation that is fuelled by plasmonic oscillations [2].

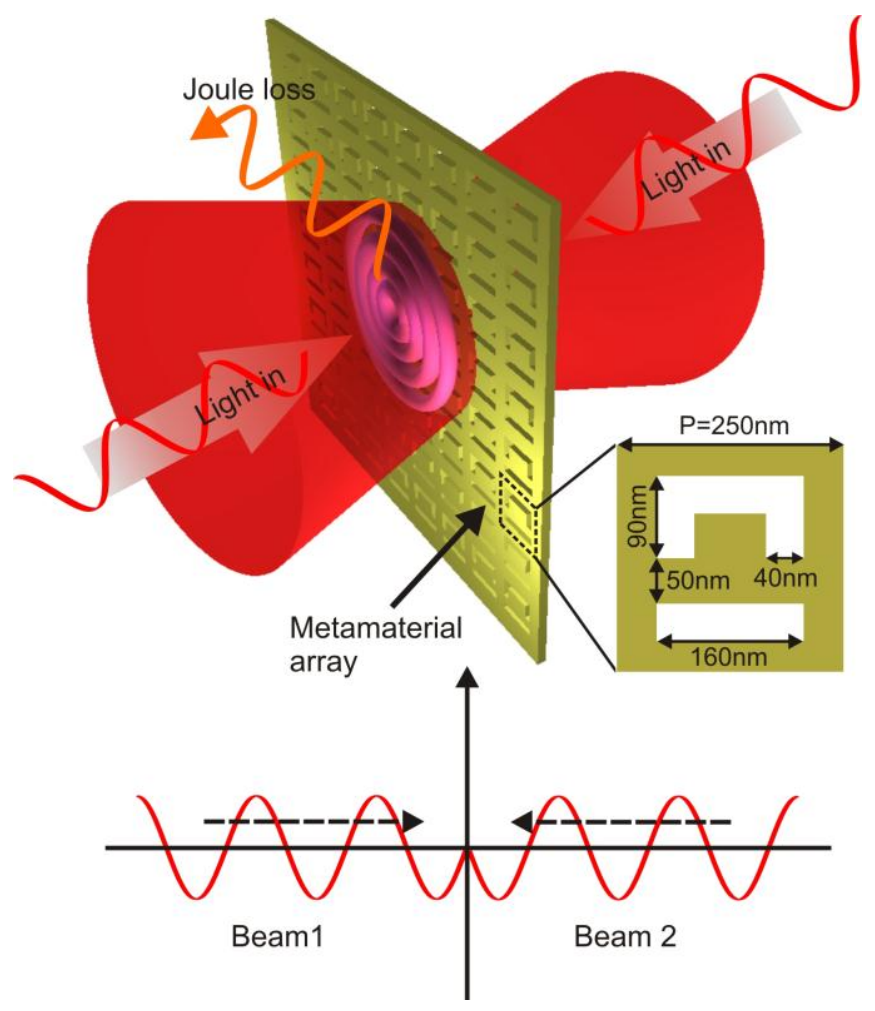

Fig. 1: (a) Artistic impression of a metamaterial coherent perfect absorber: Up to $100 \%$ of the total energy of two counter-propagating incident beams may be absorbed in a nanostructured metallic film of sub-wavelength thickness. 
In a lasing spaser, a plasmonic metamaterial dictates the wavelength at which the device emits light, with energy being drawn from a supporting gain substrate. The emission wavelength is controlled via meta-molecule design and no external resonator is required. To create a time-reversed analogue to the lasing spaser we employ metamaterials comprising two dimensional arrays of asymmetric split-ring (ASR) plasmonic resonators possessed of a Fano-type plasmonic mode. No additional dissipative medium is required as the metallic metamaterial itself is absorptive through Joule losses.

Coherent metamaterial absorption has been experimentally demonstrated at a wavelength of $633 \mathrm{~nm}$ using a 50 $\mathrm{nm}$ thick gold ASR metamaterial prepared by thermal evaporation and focused ion beam milling on a glass substrate. The experimental configuration is shown in Fig. 2a: Two y-polarized beams of equal intensity (originating from a single laser source) are focused onto the metamaterial by parabolic mirrors, their mutual phase being controlled by a piezoelectrically-driven optical delay line; a single detector provides for measurement of singlebeam transmission in either direction or thy total combined transmission of both beams.
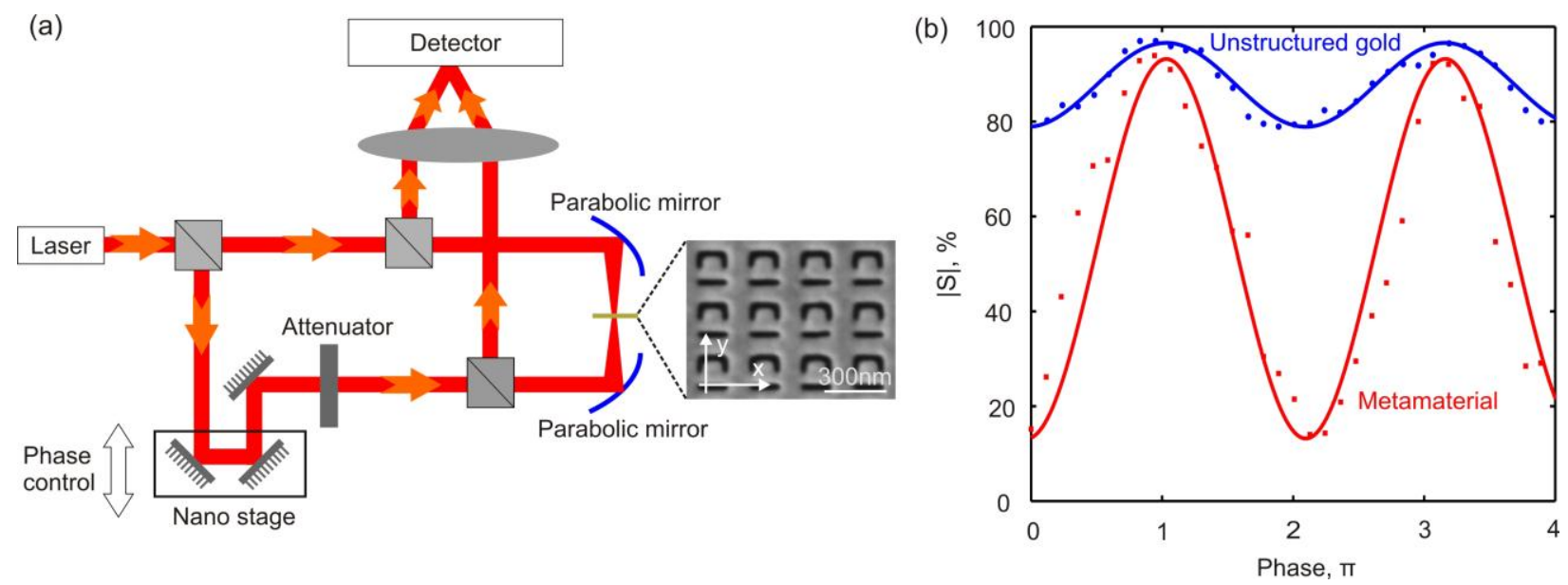

Fig. 2: (a) Experimental configuration for the demonstration of metamaterial coherent light absorption: Polarized light from a $\mathrm{He}: \mathrm{Ne}$ laser source is split into two beams that are focused from opposing sides onto a $50 \mathrm{~nm}$ thick planar gold metamaterial [a segment of which is shown in the inset secondary electron image]. A piezoelectrically-actuated delay line controls the mutual phase of the two beams; (b) Total transmitted beam intensity for the metamaterial (red) as a function of the mutual phase, and for comparison, corresponding data for an unstructured $50 \mathrm{~nm}$ thick gold film (blue).

Total transmitted intensity (Fig. 2b) is found to vary between 13\% (absorption $=87 \%$ ) when the two beams interfere constructively in the metamaterial, and 94\% (absorption $=6 \%$ ) when the interference is destructive, as a function of mutual phase. In contrast, the absorption of a $50 \mathrm{~nm}$ thick unstructured gold film changes only by about $20 \%$ between maximum and minimum (constructive and destructive interference) levels. Only the presence of the substrate and minor imperfections in sample fabrication and beam alignment preclude the experimental achievement of either total or zero absorption for the metamaterial sample.

Metamaterial coherent absorption can be implemented freely across a broad visible to near-infrared range by varying the structural design and may serve applications in optical modulators, transducers, switches or sensors.

\section{References}

[1] W. Wan, Y. Chong, L. Ge, H. Noh, A. D. Stone, and H. Cao, "Time-Reversed Lasing and Interferometric Control of Absorption," Science 331, 889-892 (2011).

[2] N. I. Zheludev, S. L. Prosvirnin, N. Papasimakis, and V. A. Fedotov, "Lasing Spaser," Nat. Photon. 2, 351-354 (2008) 